\title{
ON THE HAUSDORFF DIMENSION OF QUASICIRCLES
}

\section{J. BECKER and CH. POMMERENKE}

\section{Introduction}

For a bounded set $\Gamma$ in $C$, let $N(\varepsilon, \Gamma)$ denote the minimal number of disks of radius $\varepsilon>0$ that are needed to cover $\Gamma$. The Hausdorff dimension $[4$, p. 7$]$ satisfies

$$
\operatorname{dim} \Gamma \leqq \liminf _{\varepsilon \rightarrow 0} \frac{\log N(\varepsilon, \Gamma)}{\log (1 / \varepsilon)} .
$$

For sufficiently regular compact sets, the limit exists and is equal to $\operatorname{dim} \Gamma$, for instance if $\Gamma$ is a self-similar fractal curve [8, p. 736], [11, p. 29].

We shall study the case that $\Gamma$ is a $K$-quasicircle where $1 \leqq K<\infty$. This means that $\Gamma$ is the image of the unit circle under a $K$-quasiconformal mapping of $C$. Let $c_{1}, c_{2}, \ldots$ denote suitable positive absolute constants.

Theorem 1. Let $1 \leqq K<\infty$ and define

$$
\beta(K)=\sup _{\Gamma} \limsup _{\varepsilon \rightarrow 0} \frac{\log N(\varepsilon, \Gamma)}{\log (1 / \varepsilon)}
$$

where $\Gamma$ ranges over all $K$-quasicircles in $C$. Then

$$
\beta(K) \leqq 2-c_{1} K^{-3.41}
$$

and furthermore, for $K$ close to 1 ,

$$
1+0.36 x^{2} \leqq \beta(K) \leqq 1+37 x^{2}, \quad x=\frac{K-1}{K+1} .
$$

It follows from (1.1) and (1.2) that

$$
\operatorname{dim} \Gamma \leqq \beta(K)
$$

for all $K$-quasicircles. Gehring and Väisälä [5] were the first to show that $\beta(K)<2$. If Lehto's form [9] of the Bojarski integrability theorem is used in their proof [5, p. 507/508] one obtains

$$
\beta(K) \leqq 2-c_{2} K^{-c}
$$


with some unspecified $c \geqq 1$. It has been conjectured that $c=1$ which would be best possible.

The lower estimate in (1.4) follows from Ruelle's asymptotic expansion [17, p. 107]

$$
\operatorname{dim} J(\lambda)=1+\frac{|\lambda|^{2}}{4 \log 2}+O\left(|\lambda|^{3}\right) \quad(\lambda \rightarrow 0),
$$

where $J(\lambda)$ is the Julia set of the polynomial $w^{2}+\lambda w$.

\section{Univalent functions with quasiconformal extension}

Let $\Sigma$ denote the family of all functions

$$
g(z)=z+b_{0}+b_{1} z^{-1}+\ldots
$$

that are analytic and univalent in $\{1<|z|<\infty\}$. For $1 \leqq K<\infty$, let $\Sigma_{K}$ denote the subfamily of functions that have a $K$-quasiconformal extension to $C$. We shall deduce Theorem 1 from the following result.

Theorem 2. Let $g \in \Sigma_{K}$ and $1<r<\infty$. Then

holds with

$$
\int_{0}^{2 \pi}\left|g^{\prime}\left(r e^{i t}\right)\right|^{p} d t \leqq \frac{c_{1}}{(1-1 / r)^{p-1}}
$$

If $1 \leqq K<1+c_{3}$ then (2.2) also holds with

$$
p=2-c_{2} K^{-a}, \quad a=1+\frac{2}{\pi} \arctan 2<1.705 .
$$

It seems probable that any $a>1$ can be chosen in (2.3). Our proof would, with suitable modifications, give this if, for $g \in \Sigma$,

$$
\int_{0}^{2 \pi}\left|\exp \left[2 e^{i \theta} \log g^{\prime}\left(r e^{i t}\right)\right]\right| d t \leqq c(\varepsilon)(1-1 / r)^{-1-\varepsilon} \quad(1<r<2)
$$

is true for real $\theta$ and each $\varepsilon>0$, with $c(\varepsilon)$ depending only on $\varepsilon$. This is a strengthened version of the Brennan conjecture [2], [15].

We need the following important result [10, p. 69].

Theorem A. Let $g \in \Sigma_{K}$. Then there are functions

$$
g_{\lambda} \in \Sigma, \quad|\lambda|<1
$$

that depend analytically on $\lambda$ such that

$$
g_{0}(z)=z, \quad g_{\varkappa}=g, \quad x=(K-1) /(K+1) .
$$


Proof of Theorem 2. (a) Let

$$
\varphi(\lambda)=a_{-1} \lambda^{-1}+a_{0}+a_{1} \lambda+\ldots, \quad \varphi(1)=2, \quad a_{-1}>0
$$

map $\{|\lambda|<1\}$ conformally onto the outer domain of the triangle $\Delta$ with the vertices $2,1+i / 2$ and $1-i / 2$. We write

$$
\log g_{\lambda}^{\prime}=u_{\lambda}+i v_{\lambda}, \quad z=r e^{i t} .
$$

The function $\varphi(\lambda) \log g_{\lambda}^{\prime}(z)$ is analytic in $\{|\lambda|<1\}$, by (2.6) and (2.7). Hence

$$
\psi(\lambda)=\frac{1}{2 \pi} \int_{0}^{2 \pi}\left|g_{\lambda}^{\prime}(z)^{\varphi(\lambda)}\right| d t=\frac{1}{2 \pi} \int_{0}^{2 \pi} e^{u_{\lambda} \operatorname{Re} \varphi-v_{\lambda} \operatorname{Im} \varphi} d t
$$

is subharmonic in $\{|\lambda|<1\}$ for fixed $r>1$.

Since $g_{\lambda} \in \Sigma$, an inequality of Golusin [14, p. 65] shows that $\left|v_{\lambda}(z)\right|<$ $\log 1 /\left(1-r^{-1}\right)$ for $|\lambda|<1$. Hence (2.9) shows that

Since $[14$, p. 127]

$$
\psi(\lambda) \leqq(1-1 / r)^{-|\operatorname{Im} \varphi(\lambda)|} \frac{1}{2 \pi} \int_{0}^{2 \pi} e^{u_{\lambda} \operatorname{Re} \varphi} d t .
$$

$$
\frac{1}{2 \pi} \int_{0}^{2 \pi} c^{2 u_{\lambda}(z)} d t=\frac{1}{2 \pi} \int_{0}^{2 \pi}\left|g_{\lambda}^{\prime}(z)\right|^{2} d t<c_{4}(1-1 / r)^{-1}
$$

we therefore obtain that

$$
\psi(\lambda) \leqq\left(c_{4}(1-1 / r)^{-1}\right)^{\operatorname{Re} \varphi(\lambda) / 2}(1-1 / r)^{-|\operatorname{Im} \varphi(\lambda)|}
$$

if $|\lambda|<1$ and $0<\operatorname{Re} \varphi(\lambda) \leqq 2$. The choice of the triangle $\Delta$ shows that

$$
\operatorname{Re} \varphi(\lambda) / 2+|\operatorname{Im} \varphi(\lambda)| \leqq 1 \text { for }|\lambda|=1 .
$$

Hence we obtain from (2.10) by the maximum principle for subharmonic functions that

$$
\psi(\lambda) \leqq c_{5}(1-1 / r)^{-1} \text { for }|\lambda|<1 .
$$

Since $\varphi(1)=2$ by (2.7), it follows from the Schwarz-Christoffel formula that

$$
\varphi^{\prime}(\lambda)=-a_{-1} \lambda^{-2}(1-\lambda)^{\alpha}\left(1-\bar{\lambda}_{1} \lambda\right)^{\alpha_{1}}\left(1-\lambda_{1} \lambda\right)^{\alpha_{1}}
$$

with $\left|\lambda_{1}\right|=1$ and $\alpha=(2 / \pi)$ arc $\tan 2$ and therefore

$$
\varphi(\lambda)=2+c_{6}(1-\lambda)^{\alpha+1}+o\left((1-\lambda)^{\alpha+1}\right) \quad \text { as } \quad \lambda \rightarrow 1-0 .
$$

Hence (2.3) holds if we set $p=\varphi(x) /(\varphi(x)-1)$. Furthermore, by (2.6) and (2.9),

$$
\left(\frac{1}{2 \pi} \int_{0}^{2 \pi}\left|g^{\prime}\right|^{p} d t\right)^{1 / p} \leqq\left(\frac{1}{2 \pi} \int_{0}^{2 \pi}\left|g^{\prime}\right|^{\varphi(x)} d t\right)^{1 / \varphi(\varkappa)}=\psi(\varkappa)^{1 / \varphi(x)}
$$

and (2.11) therefore shows that

$$
\int_{0}^{2 \pi}\left|g^{\prime}(z)\right|^{p} d t \leqq c_{1}(1-1 / r)^{-p / \varphi(x)}=c_{1}(1-1 / r)^{-p+1} .
$$


(b) Since $g \in \Sigma_{K}$ it follows from Golusin's estimate [7, p, 132] for the class $\Sigma$ and from Theorem A by Lehto's majorant principle that

$$
\left|\frac{g^{\prime \prime}(z)}{g^{\prime}(z)}\right| \leqq \frac{6 \varkappa}{r^{2}-1} \leqq \frac{3 \varkappa}{r-1} \quad \text { for } \quad z=r e^{i t}, \quad r>1 .
$$

We conclude as in [13] or [6] that

$$
\int_{0}^{2 \pi}\left|g^{\prime}(z)\right|^{p} d t \leqq c_{7}(1-1 / r)^{-9 p^{2} x^{2}}
$$

and this implies (2.2) with $p$ given by (2.4) because $9 p^{2} \varkappa^{2} \leqq p-1$ if $0 \leqq \varkappa<c_{8}$.

\section{Proof of Theorem 1}

(i) Let $\Gamma$ be a $K$-quasicircle in $C$. Then there is a $K$-quasiconformal mapping $w$ of $\hat{\boldsymbol{C}}$ mapping $\{|z|=1\}$ onto $\Gamma$. Let $g$ be a conformal mapping of $\{|z|>1\}$ onto the outer domain of $\Gamma$; we may assume that $g$ has the form (2.1). If we define

$$
g(z)=w\left(1 \overline{w^{-1} \circ g(1 / \bar{z})}\right) \text { for }|z| \leqq 1
$$

then $g \in \Sigma_{K^{*}}$ with $K^{*}=K^{2}$; see e.g. [10, p. 39/40].

It follows from Theorem 2 that (2.2) holds with

$$
p=2-c_{2} K^{-2 a}
$$

and, for $K$ close to 1 , also with

$$
p=1+9.1 \varkappa^{* 2} \leqq 1+37 x^{2} .
$$

Hence our upper estimates are an immediate consequence of the following result which is the analogue of [16, Corollary 2] for the class $\Sigma$.

Theorem B. Let $g \in \Sigma$. If $\{g(z):|z|>1\}$ is bounded by a quasicircle $\Gamma$ and if

$$
\int_{0}^{2 \pi}\left|g^{\prime}\left(r e^{i t}\right)\right|^{p} d t=O\left(\frac{1}{(1-1 / r)^{p-1}}\right) \quad \text { as } \quad r \rightarrow 1+0
$$

then

$$
\limsup _{\varepsilon \rightarrow 0} \frac{\log N(\varepsilon, \Gamma)}{\log (1 / \varepsilon)} \leqq p .
$$

(ii) We need some known facts from complex iteration theory to prove the lower estimate; see e.g. [3] or [12] for an overview. We give a brief indication of the proofs.

Let $|\lambda|<1$ and let $J(\lambda)$ be the Julia set associated with $w^{2}+\lambda w$; this is a Jordan curve. If

$$
g_{\lambda}(z)=b_{-1}(\lambda) z+\sum_{n=0}^{\infty} b_{n}(\lambda) z^{-n}
$$


maps $\{|z|>1\}$ conformally onto the outer domain of $J(\lambda)$, then

$$
g_{\lambda}\left(z^{2}\right)=g_{\lambda}(z)^{2}+\lambda g_{\lambda}(z) \text { for }|z|>1 \text {. }
$$

It follows that $b_{-1}(\lambda)=1$ and, by induction, that $b_{n}(\lambda)$ is a polynomial in $\lambda$. Since $\left|b_{n}(\lambda)\right|<1$ for $n \geqq 1$ by the area theorem, we conclude that $g_{\lambda}$ depends analytically on $\lambda$.

Since $g_{\lambda}(z)$ is univalent in $\{|z|>1\}$ and since $g_{0}(z)=z$, a deep result of Bers and Royden [1, Theorem 1] shows that

$$
g_{\lambda} \in \Sigma_{K}, \quad x=|\lambda| .
$$

Hence $J(\lambda)$ is a $K$-quasicircle, and $\beta(K) \geqq 1+0.36 x^{2}$ (for $K$ close to 1 ) follows from (1.1), (1.2) and Ruelle's estimate (1.6).

\section{References}

[1] Bers, L., and H. L. RoYden: Holomorphic families of injections - Acta Math. 157, 1986, $259-286$.

[2] BRennan, J. E.: The integrability of the derivative in conformal mapping. - J. London Math. Soc. (2) $18,1973,261-272$.

[3] BRolin, H.: Invariant sets under iteration of rational functions. - Ark. Mat. 6, 1965, 103-144.

[4] FALCONER, K. J.: The geometry of fractal sets. - Cambridge University Press, 1985.

[5] GehrING, F. W., and J. VÄISÄLÄ: Hausdorff dimension and quasiconformal mapping. - J. London Math. Soc. (2) 6, 1973, 504-512.

[6] GökтüRK, Z.: Estimates for univalent functions with quasiconformal extensions. - Ann. Acad. Sci. Fenn. Ser. A I Math. 589, 1974, 1-21.

[7] Golusin, G. M.: Geometrische Funktionentheorie. - VEB Deutscher Verlag der Wissenschaften, Berlin, 1957.

[8] Hutchinson, J. E.: Fractals and self similarity. - Indiana Univ. Math. J. 30, 1981, 713-747.

[9] LeHTo, O.: Remarks on the integrability of the derivatives of quasiconformal mappings. - Ann. Acad. Sci. Fenn. Ser. A I Math. 371, 1965, 1-8.

[10] Lehto, O.: Univalent functions and Teichmüller spaces. - Springer-Verlag, New York, 1987.

[11] Martio, O., and M. Vuorinen: Whitney cubes, $p$-capacity, and Minkowski content. - Exposition Math. 5, 1987, 17-40.

[12] Peitgen, H.-O., and P. H. Richter: The beauty of fractals. - Springer-Verlag, Berlin, 1986.

[13] Pommerenke, CH.: On the growth of the coefficients of analytic functions. - J. London Math. Soc. (2) 5, 1972, 624-628.

[14] Pommerenke, Сн.: Univalent functions. - Vandenhoeck \& Ruprecht, Göttingen, 1975.

[15] Pommerenke, Сн.: On the integral means of the derivative of a univalent function, II. - Bull. London Math. Soc. 17, 1985, 565-570.

[16] Pommerenke, Сh.: On boundary size and conformal mapping. - Preprint TU Berlin, 1987.

[17] Ruelle, D.: Repellers for real analytic maps. - Ergodic Theory Dynamical Systems 2, 1982, 99-107.

Technische Universität Berlin

Fachbereich Mathematik

D 1000 Berlin 12

Bundesrepublik Deutschland

Received 20 May 1987 